\title{
Crecimiento en diámetro de tres especies arbóreas en una selva secundaria de Quintana Roo, México
}

\section{Diameter growth of the three tree species in a secondary forest of Quintana Roo, Mexico}

\author{
Elizandro Pineda-Herreral", Roy Jabín Carreón-Santos², Juan Ignacio Valdez-Hernández² y Víctor Manuel Interián-Ku³ \\ Instituto Potosino de Investigación Científica y \\ Tecnológica, A.C. División de Ciencias Ambientales. \\ San Luis Potosí, San Luis Potosí, México. \\ 2 Colegio de Postgraduados. Campus Montecillo. \\ Postgrado Forestal, Texcoco, Estado de México \\ México. \\ 3 Instituto Tecnológico de la Zona Maya. Chetumal, \\ Quintana Roo, México. \\ * Autor de correspondencia. \\ elherrera2001@yahoo.com.mx
}

\section{RESUMEN}

Bursera simaruba, Lysiloma latisiliquum y Piscidia piscipula son tres especies de las selvas subperennifolias de Quintana Roo que son valoradas favorablemente por su madera. Para seguir incentivando su aprovechamiento y conservación, así como su conocimiento ecológico, se requiere determinar la tasa de crecimiento anual y los factores que lo modulan. En el presente estudio se midió el crecimiento en diámetro de Bursera simaruba, Lysiloma latisiliquum y Piscidia piscipula, en selvas secundarias medianas subperennifolias del ejido Andrés Quintana Roo, Quintana Roo. Setenta y tres árboles fueron categorizados en cinco intervalos de diámetro y medidos cada dos meses, empleando bandas dendrométricas; el estudio abarcó desde enero de 2011 hasta mayo de 2012. Los incrementos se correlacionaron con la precipitación, temperatura promedio del aire y humedad relativa locales. Los incrementos se ajustaron a un modelo polinómico de tercer orden. $L$. latisiliquum presentó la tasa de crecimiento promedio anual más alta $(0.18 \mathrm{~cm})$, seguida por $P$. piscipula $(0.10 \mathrm{~cm})$ y por B. simaruba $(0.06$ $\mathrm{cm})$. Las categorías diamétricas de las tres especies presentaron diferencias significativas en sus incrementos; las de $20 \mathrm{~cm}$, de forma general, crecieron más que las restantes. Los incrementos se correlacionaron de forma significativa $(p \leq 0.01)$ con la temperatura en las tres especies; la precipitación y la humedad relativa no tuvieron una influencia marcada en el engrosamiento del fuste. La presente investigación demostró tres patrones distintos de crecimiento, distinguiendo a la temperatura como un factor de influencia en el aumento del diámetro.

PALABRAS CLAVE: bandas dendrometricas; Bursera simaruba; Lysiloma latisiliquum; Piscidia piscipula; temperatura.

\section{ABSTRACT}

Bursera simaruba, Lysiloma latisiliquum and Piscidia piscipula are three species of the semi-evergreen tropical forests of Quintana Roo that are valued for their wood. To continue encouraging their use and conservation, as well as their ecological knowledge, it is necessary to determine the annual growth rate and the factors that modulate it. In the present study, the diameter growth of B. simaruba, L. latisiliquum and P. piscipula was measured in a semi-evergreen secondary tropical forest of the Andrés Quintana Roo ejido, Quintana Roo. 73 trees were categorized into five diameter intervals and measured every two months using dendrometric bands. The study ran from January 2011 to May 2012. The increases were correlated with precipitation, average air temperature and local relative humidity. The increases were adjusted to a third-order polynomial model. L. latisiliquum had the highest annual average growth rate $(0.18 \mathrm{~cm})$, followed by $P$. piscipula $(0.10 \mathrm{~cm})$ and B. simaruba $(0.06 \mathrm{~cm})$. The diametric categories of the three species showed significant differences in their increments, those of $20 \mathrm{~cm}$ in general grew more than the rest. The increases correlated significantly $(p<0.01)$ with the temperature in the three species, precipitation and relative humidity did not have a marked influence on the thickening of the bole. The present investigation showed three different patterns of growth, distinguishing temperature as an influencing factor in the increase in diameter.

KEYWORDS: dendrometrics bands; Bursera simaruba; Lysiloma latisiliqum; Piscidia piscipula; temperature. 


\section{INTRODUCCIÓN}

El estado de Quintana Roo cuenta con selvas bajas caducifolias, medianas subcaducifolias y subperennifolias (Miranda y Hernández, 1963; Pennington y Sarukhán, 2005), en las cuales se realiza silvicultura desde hace aproximadamente 60 años (Negreros-Castillo et al., 2014). Se estima que el aprovechamiento forestal de estas selvas además de las reservas naturales son los factores que permiten la permanencia y ganancia de superficie arbolada en el estado (Ellis, Hernández y Romero-Montero, 2017), por lo que se debe procurar su continuidad de manera adecuada y sostenible (Negreros-Castillo y Mize, 2013).

La caoba (Swietenia macrophylla King) y el cedro (Cedrela odorata L.) son las especies más valoradas en Quintana Roo y el avance en el conocimiento de su ecología y silvicultura ha sido sustancial (Snook, 2005; Negreros-Castillo y Mize, 2013; Negreros-Castillo et al., 2014). Bursera simaruba (L.) Sarg., Lysiloma latisiliquum (L.) (Benth.) y Piscidia piscipula (L.) Sarg. son otras especies aprovechadas en el estado, cuyos volúmenes maderables llegan a ser tan importantes como los de caoba y cedro, por lo que es idóneo ampliar el conocimiento ecológico y técnico sobre ellas para conservar la composición, la estructura y el funcionamiento de las selvas en que se desarrollan (Negreros-Castillo y MartínezSalazar, 2011; Negreros-Castillo et al., 2014).

Para aprovechar cualquier especie leñosa es necesario conocer su tasa de crecimiento, así como los múltiples factores que en ella inciden, por ejemplo: el ambiente, la fisiología o la morfología. Estimar el crecimiento también tiene implicaciones en: prospección del cambio climático (Way y Oren, 2010), competencia ecológica (O’Connor, Lynch, Falk y Swetnam, 2015), captura de carbono (Vanderwel, Zeng, Caspersen, Kunstler y Lichstein, 2016), dinámica de poblaciones (Fassnacht et al., 2015) y restauración ecológica (Fahey y Lorimer, 2013).

En la península de Yucatán los antecedentes de crecimiento sobre estas especies son los trabajos de Mize, Centeno y Negreros-Castillo (1997), en selvas subperennifolias de Campeche, que concluyen que los incrementos de fuste no fueron diferentes entre tres tipos de suelo (Litosol, Rendzina, Vertisol) ni entre edades del bosque (18 años, 24 años, 29 años y 30 años). Para $L$. latisiliquum las investigaciones de López-Torres y TamaritUrias (2005), también en Campeche, así como las de García y Rodríguez (1993) y Negreros-Castillo y Martínez-Salazar (2011) en Quintana Roo mencionan que la apertura del dosel y cortas intermedias de mejoramiento inducen mayores incrementos en diámetro.

La complejidad del proceso del crecimiento vegetal no puede adjudicarse únicamente a una variable, sino a una serie de factores abióticos y bióticos (Baker, Swaine y Burslem, 2003). Algunos estudios señalan que el clima ocuparía el primer lugar, las características del micrositio el segundo $y$, por último, la naturaleza e intensidad del disturbio que regeneró al bosque (Toledo et al., 2011). Sin embargo, la estructura, la composición de otras selvas y las características bioecológicas de cada especie no permitirían extrapolar este orden.

Pocos estudios han establecido la relación climacrecimiento en la Península de Yucatán (Interián-Ku, Vaquera-Huerta, Valdez-Hernández, García-Moya y Romero-Manzanares, 2014; Estrada-Medina, Santiago, Graham, Allen y Jiménez-Osornio, 2013) y los resultados indican que el elemento precipitación ocupa un lugar primario en este proceso. Investigaciones de mayor profundidad sobre la temperatura, la humedad, la radiación solar, etc., sobre el crecimiento arbóreo son incipientes en México (Yáñez-Espinosa, Terrazas y López-Mata, 2006; Ricker, Gutiérrez-García y Daly, 2007). La importancia de incluir a la temperatura y/o a la humedad radica en que intervienen en el inicio de la foliación y la consecuente activación de la actividad del cambium (Lloyd y Farquhar, 2008; Way y Oren, 2010).

Por lo anterior, es probable que el crecimiento de otras especies de las selvas de Quintana Roo esté relacionado no únicamente con la lluvia y su distribución, sino que otros elementos pueden tener una influencia de la misma o mayor magnitud.

\section{OBJETIVOS}

Determinar el crecimiento en diámetro de Bursera simaruba, Lysiloma latisiliquum y Piscidia piscipula en una selva 
secundaria subperennifolia y correlacionarlo con la precipitación, la temperatura y la humedad relativa

\section{MATERIALES Y MÉTODOS}

\section{Área de estudio}

El sitio de estudio se localiza en el ejido Andrés Quintana Roo del municipio Felipe Carrillo Puerto, Quintana Roo (1958' 5.2” N, $87^{\circ} 53$ ' 12.2” O). Geomorfológicamente es una planicie caracterizada por bancos de yeso de varios metros de espesor intercalados entre rocas calizas cristalinas y margas blancas o amarillas (Pozo, Armijo y Calmé, 2011). Los suelos, según la clasificación maya, son los denominados tzequel (litosoles) con una profundidad menor de $15 \mathrm{~cm}$ con afloración de rocas de color negro con presencia intercalada de yaaxhom (vertisoles gleicos o gleisoles) de profundidad variable y de color negro a gris y kankab (rendzinas) de color rojo a negro con profundidades variables (Organización de Ejidos Productores Forestales de la Zona Maya, S.C. [Oepfzm], 2000; Organización Mundial de las Naciones Unidas para la Agrigultura y la Alimentación [FAO], 2007). El clima es de tipo Aw 1 , cálido subhúmedo con lluvias en verano, con temperatura media anual de $25.8^{\circ} \mathrm{C}$ y una precipitación media anual de 1204.5 mm (García, 1987).

El tipo de vegetación es selva mediana subperennifolia que conserva de $50 \%$ a $75 \%$ su follaje durante la época seca, con algunas lluvias en la temporada seca (Miranda y Hernández, 1963). La altura del dosel varía de $15 \mathrm{~m}$ a $25 \mathrm{~m}$ dependiendo del tipo de suelo y especie arbórea presente. Los árboles dominantes son: Metopium Browne Jacq. Urb., Manilkara zapota (L.) P. Royen, Bursera simaruba (L.) Sarg. y Lysiloma latisiliquum (L.) (Benth.); otras arbóreas: Nectandra spp. Rol. ex Rottb., Byrsonima bucidaefolia Standl., Caesalpinia gaumeri Greenm., Piscidia piscipula (L.) Sarg., Piscidium sartorianum (O. Berg) Nied., Vitex gaumer Greenm.y Beaucarnea ameliae Lem. y las palmas: Thrinax radiata Lodd. ex Schultz. \&Schult., Coccothrinax readii H. J. Quero, Pseudophoenix sargenti H. Wendl. ex Sarg. (Miranda y Hernández, 1963; Pennington y Sarukhán, 2005).

\section{Muestreo de árboles y medición del diámetro}

En dos unidades de muestreo de $1000 \mathrm{~m}^{2}(20 \mathrm{~m} \times 50 \mathrm{~m})$, ambos con una temporalidad de sucesión de 30 años y cuyo uso anterior fue el ganadero, se eligieron: 73 árboles (> 2.5 cm DAP): 23 de Bursera simaruba, 25 de Lysiloma latisiliquum y 25 de Piscidia piscipula. Todos los individuos seleccionados presentaron fuste recto. Fueron clasificados por categoría de diámetro: $5=2.5 \mathrm{~cm}-7.49 \mathrm{~cm} ; 10=7.5 \mathrm{~cm}-12.49 \mathrm{~cm}$; $15=12.5 \mathrm{~cm}-17.49 \mathrm{~cm} ; 20=17.5 \mathrm{~cm}-22.49 \mathrm{~cm}$ (LópezAyala, Valdez-Hernández, Terrazas y Valdez-Lazalde, 2006).

A todos los árboles se les colocaron bandas dendrométricas de acero inoxidable fabricadas en el lugar (Cattelino, Becher y Fuller, 1986). De enero de 2010 a mayo de 2011 se realizaron medidas bimestrales.

La precipitación, temperatura promedio y la humedad relativa (Tabla 1) para el periodo de medición se obtuvieron de la estación de Felipe Carrillo Puerto $\left(19^{\circ} 34^{\prime} 32^{\prime \prime}\right.$ N, $88^{\circ}$ 02’ 42” O) del Servicio Meteorológico Nacional.

TABLA 1. Precipitación, temperatura y humedad relativa para la zona de estudio durante el periodo de medición

\begin{tabular}{lccc}
\hline Fecha & $\begin{array}{c}\text { Precipitación } \\
(\mathrm{mm})\end{array}$ & $\begin{array}{c}\text { Temperatura } \\
\left({ }^{\circ} \mathrm{C}\right)\end{array}$ & $\begin{array}{c}\text { Humedad } \\
\text { relativa }(\%)\end{array}$ \\
\hline enero 2010 & 19.79 & 22.17 & 79.96 \\
marzo 2010 & 10.4 & 23.82 & 71.71 \\
mayo20 10 & 345.13 & 28.27 & 82.64 \\
julio 2010 & 392.5 & 27.38 & 91.1 \\
septiembre 2010 & 124.38 & 27.62 & 88.68 \\
noviembre 2010 & 11.18 & 23.73 & 82.27 \\
enero 2011 & 31.98 & 22.89 & 84.17 \\
marzo 2011 & 122.4 & 24.94 & 80.22 \\
mayo 2011 & 1.77 & 28.74 & 74.97 \\
\hline
\end{tabular}

\section{Ecuaciones de crecimiento}

Para modelar el crecimiento acumulado en función del tiempo, se empleó el siguiente modelo polinomial de tercer orden (Kiviste, González, Alboreca, y González, 2010):

$$
y=b_{0}+b_{1} x+b_{2} x^{2}+b_{3} x^{3}
$$


La ecuación fue evaluada a través de técnicas de regresión no lineal en el programa Curve Expert (Hyams, 2005). Se empleó el método de mínimos cuadrados generalizados, en los cuales se comprobaron los supuestos de normalidad y homogeneidad de varianzas (Guerra, Cabrera y Fernández, 2003). Los indicadores de ajuste fueron: cuadrado medio del error (CME), coeficiente de determinación $\left(\mathrm{R}^{2}\right)$, y nivel de significancia estadística $(p \leq 0.01)$ de los parámetros de cada ecuación (Guerra et al., 2003).

\section{Análisis estadístico}

Para comparar el crecimiento entre categorías diamétricas se realizó un análisis de varianza (ANDEVA) (Gotelli y Ellison, 2004) y una prueba de comparación múltiple (Bonferroni) (Gotelli y Ellison, 2004). La precipitación, la temperatura y la humedad relativa se correlacionaron con los incrementos en diámetro (Pearson) (Gotelli y Ellison, 2004). El paquete estadístico empleado fue Stata versión 9.1 (Stata Corp, 2001).

\section{Resultados}

Las curvas de incremento general de las tres especies mostraron valores altos de R2 y niveles de confianza estadística aceptables. Lysiloma latisiliquum fue la que tuvo mayor aumento en diámetro, seguida por Piscidia piscipula y Bursera simaruba. La curva de L. latisiliquum tuvo la mayor pendiente respecto a las otras dos. En P. piscipula se aprecianmomentos de menor incremento, lo cual se relaciona con la época de lluvias (verano de 2010). B. simaruba presentó la curva con la menor pendiente, aunque con ritmo sostenido durante todo el estudio (Fig. 1).

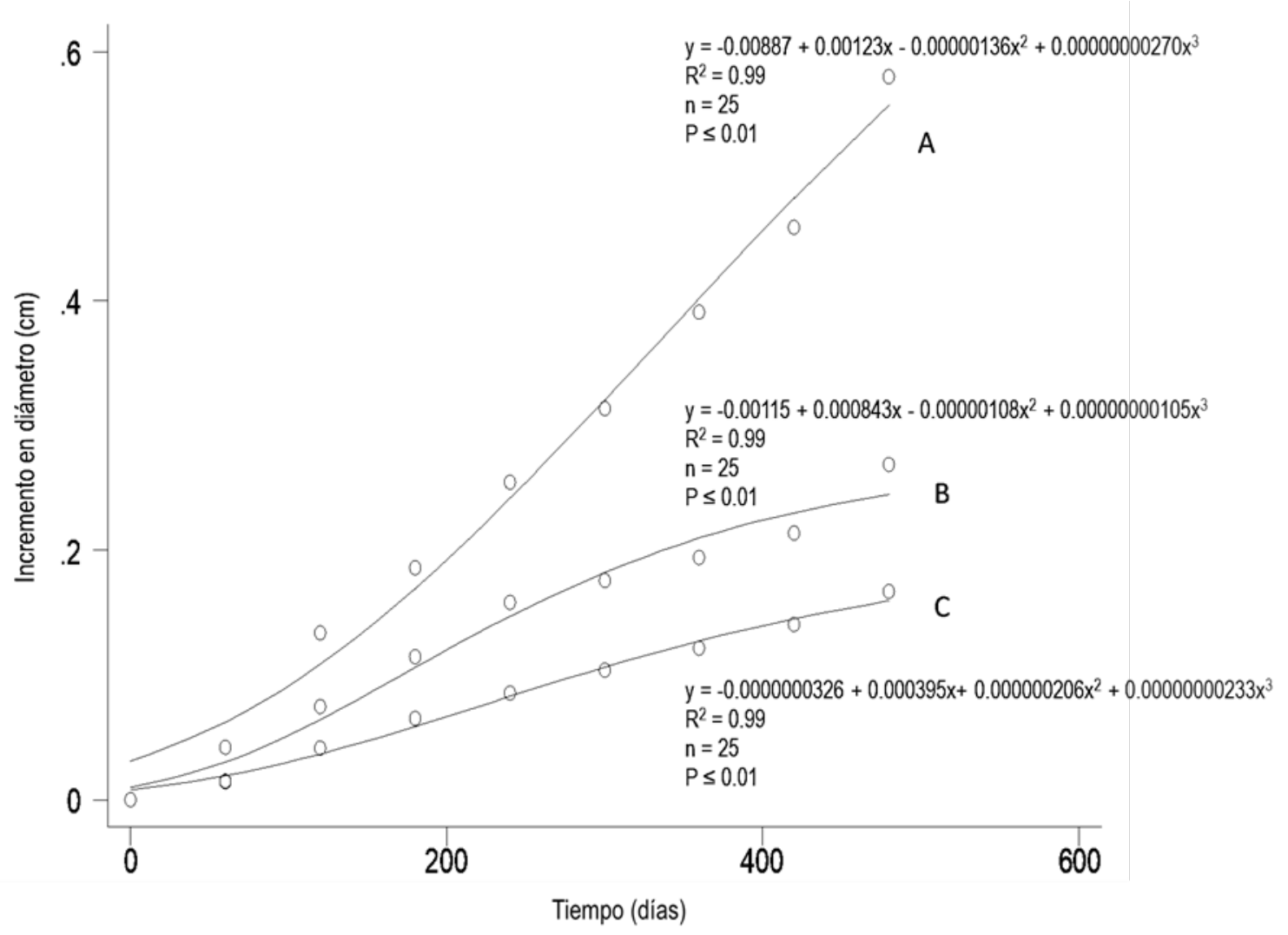

FIGURA 1. Curvas de crecimiento acumulado A. Lysiloma latisiliquum. B. Piscidia piscipula. C. Bursera simaruba. Los círculos son los incrementos promedio por cada medición, la línea representa la curva ajustada. 
La tabla 2 muestra los incrementos que presentaron las tres especies, confirmándose que Lysiloma latisiliquum fue superior en máximo, promedio y mínimo.

Entre las categorías diamétricas de Bursera simaruba, la 20 mostró los mayores incrementos, seguida de 15, 10 y 5.
En dicha categoría se presentaron los individuos con los mayores aportes de incremento en diámetro de la muestra. Los valores estadísticos del modelo mostraron valores de $\mathrm{R}^{2}$ medianos en las categorías 5 y 10 y valores bajos en en las categorías 15 y 20 (Fig. 2).

TABLA 2. Incrementos anuales, máximo, promedio, mínimo y desviación estándar de tres especies de la selva mediana subperennifolia de Quintana Roo, México.

\begin{tabular}{lcccc}
\hline Especie & \multicolumn{3}{c}{ Incremento $(\mathrm{cm})$} & Desviación estándar \\
\hline & Máximo & Promedio & Mínimo & \\
\cline { 2 - 4 } Bursera simaruba & 0.1669 & 0.0615 & 0.0137 & 0.0537 \\
Lysiloma latisiliquum & 0.5793 & 0.1884 & 0.0416 & 0.1820 \\
Piscidia piscipula & 0.2688 & 0.1044 & 0.0149 & 0.0859 \\
\hline
\end{tabular}
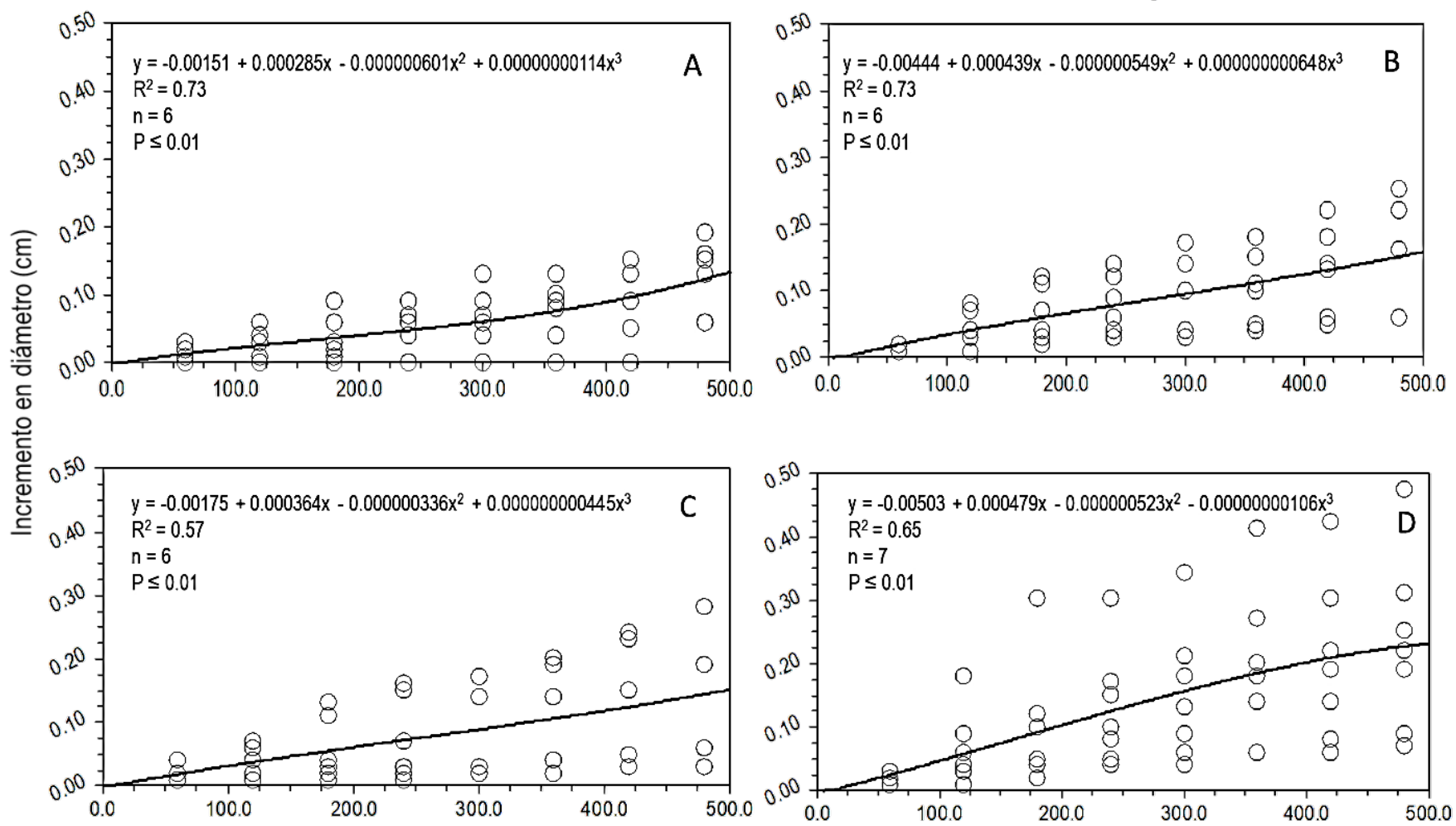

\section{Tiempo (días)}

FIGURA 2. Curvas de incremento acumulado de las categorías diámetricas de Bursera simaruba.

A = categoría 5; B = categoría 10; C = categoría 15 y D = categoría 20. Los círculos son los incrementos por cada medición de cada árbol, la línea representa la curva ajustada. 
La categoría 20 de Lysiloma latisiliquum presentó los mayores incrementos, seguida por 15, 5 y 10 . Los valores de $\mathrm{R}^{2}$ fueron bajos debido a que en las cuatro categorías se presentaron individuos con incrementos destacados en la muestra (Fig. 3).

Los incrementos en la categoría 15 fueron mayores a los de 20,10 y 5 en Piscidia piscipula. Los valores de $\mathrm{R}^{2}$ fueron medianos dado que se presentaron individuos con incrementos destacados en las categorías 15 y 20 (Fig. 4).

En la tabla 3 se muestran los incrementos promedio y totales por categoría diamétrica de las tres especies. Los incrementos de las categorías de Lysiloma latisiliquum superan a los de Piscidia piscipula y de Bursera simaruba en ese orden. La categoría con los mayores incrementos en las tres especies fue la 20 y aquellos incrementos con los menores valores correspondieron a las categorías 5, 10 y 15 para $B$. simaruba, L. latisiliquum у P. piscipula, respectivamente. El análisis de varianza, mostró que en las tres especies se encontraron diferencias significativas entre las categorías diamétricas de las tres especies, dichas variaciones fueron más significativas en Bursera simaruba, seguidas por Piscidia piscipula y por último en Lysiloma latisiliquum (Tabla 4).

Entre las categorías diamétricas de Bursera simaruba la 20 presentó mayor crecimiento que el resto. Para Lysiloma latisiliquum la diferencia se encontró entre las categorías 10 y 20. Piscidia piscipula presentó los mayores crecimientos en las categorías de 10 y 20 , mientras que los menores se dieron en las categorías 5 y 15 (Tabla 5).

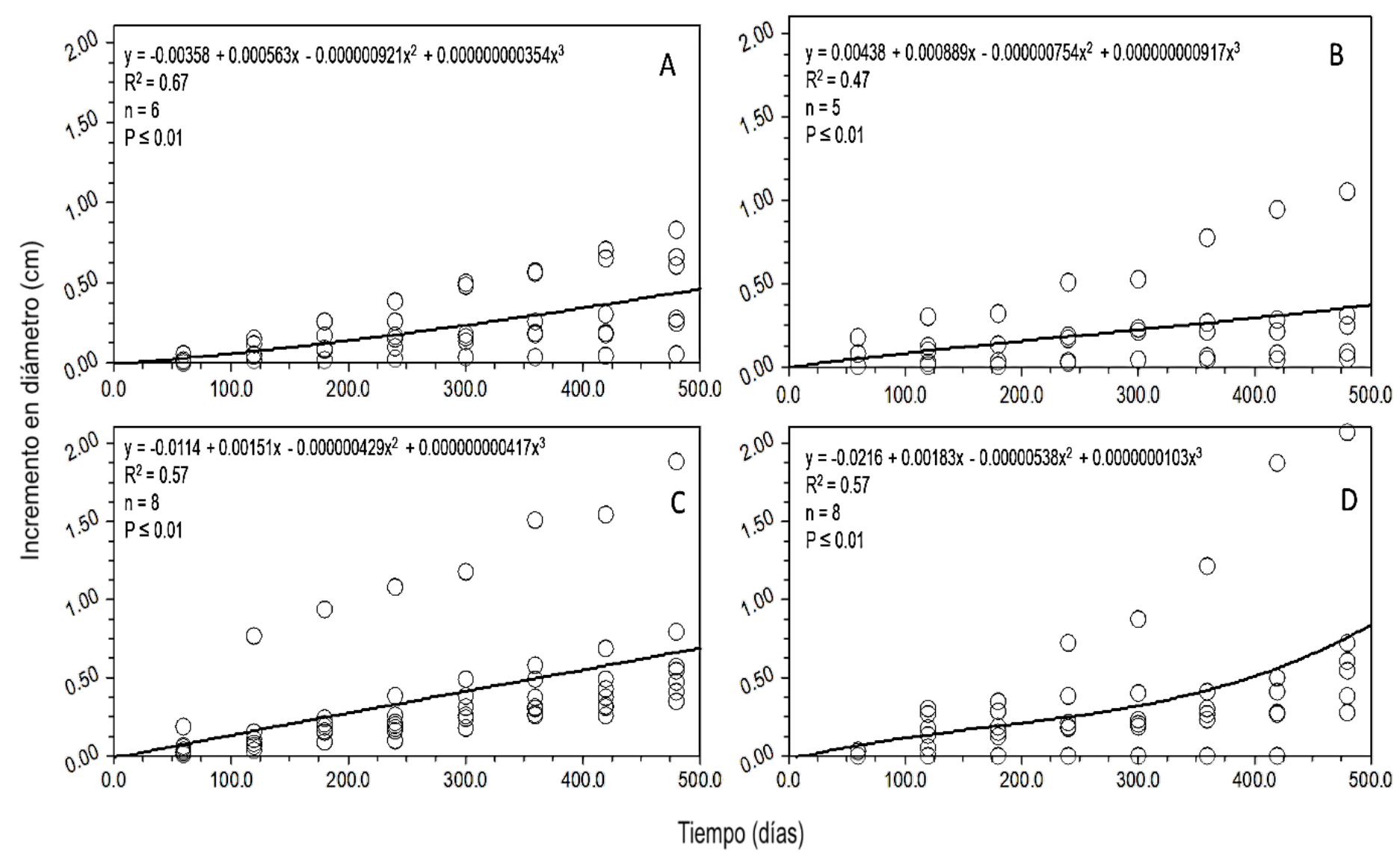

FIGURA 3. Curvas de incremento en diámetro de las categorías diámetricas de Lysiloma latisiliquum.

$A$ = categoría 5; $\mathrm{B}$ = categoría 10; C = categoría 15 y D = categoría 20. Los círculos son los incrementos por cada medición de cada árbol, la línea representa la curva ajustada. 

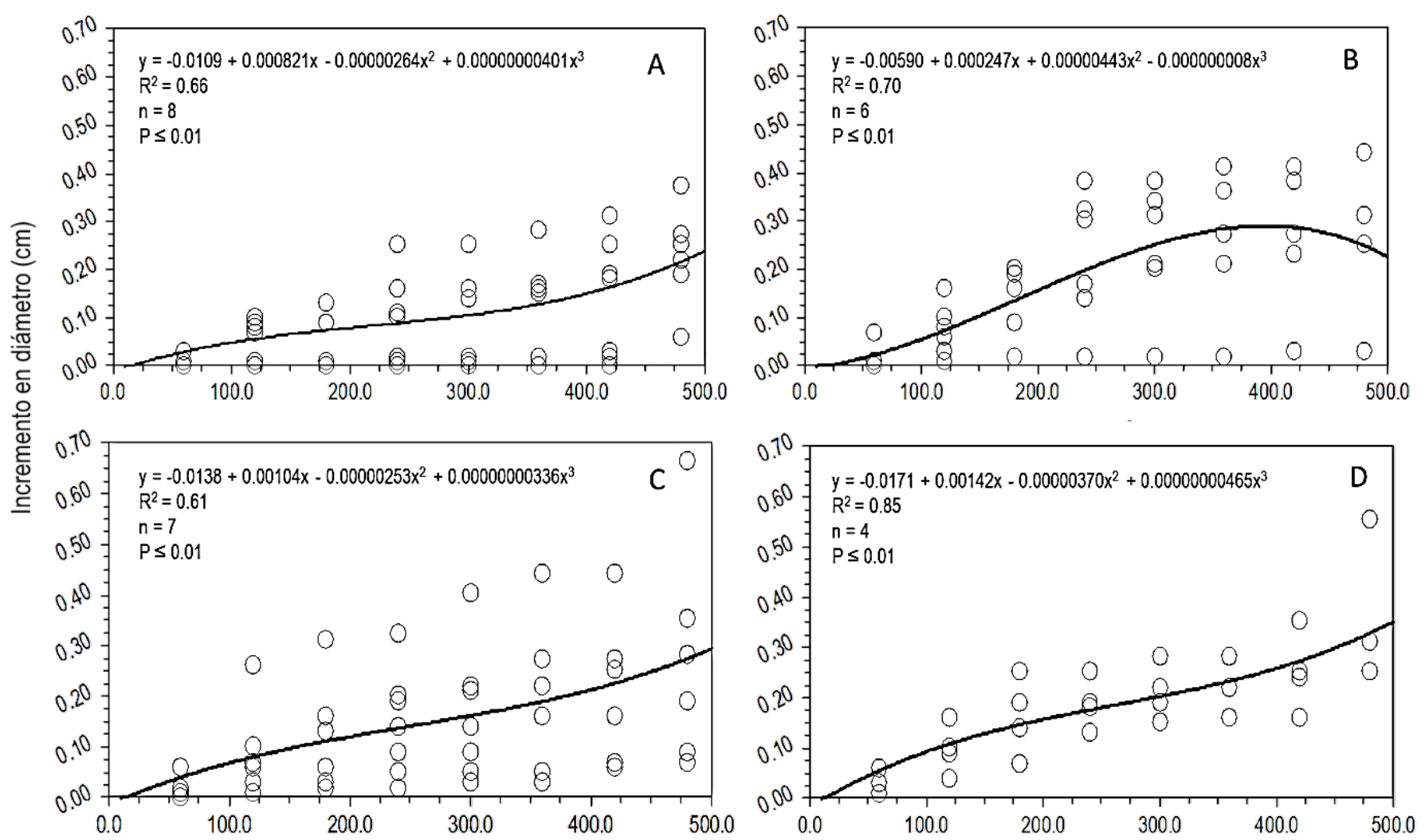

Tiempo (dias)

FIGURA 4. Curvas de incremento en diámetro de las categorías diámetricas de Piscidia piscipula.

A = categoría $5 ; \mathrm{B}$ = categoría $10 ; C$ = categoría 15 y D = categoría 20. Los círculos son los incrementos por cada medición de cada árbol, la línea representa la curva ajustada.

TABLA 3. Incrementos en diámetro por categoría diamétrica de tres especies de la selva mediana subperennifolia de Quintana Roo, México.

\begin{tabular}{lccccc}
\hline Especie & \multicolumn{5}{c}{ Categoría diamétrica $(\mathrm{cm})$} \\
\hline Bursera simaruba & 5 & 10 & 15 & 20 & Promedio \\
Lysiloma latisiliquum & 0.0535 & 0.0764 & 0.0720 & 0.1197 & 0.0804 \\
Piscidia piscipula & 0.1958 & 0.1848 & 0.3304 & 0.3009 & 0.2530 \\
\hline
\end{tabular}

TABLA 4. Análisis de varianza de tres especies de la selva mediana subperennifolia de Quintana Roo, México.

\begin{tabular}{llccccc}
\hline Especie & Fuente de variación & $S C$ & $G L$ & $M S$ & $F$ & Prob $>F$ \\
\hline \multirow{2}{*}{$\begin{array}{l}\text { Bursera } \\
\text { simaruba }\end{array}$} & Entre grupos & 0.140257091 & 3 & 0.046752364 & & \\
& Intragrupos & 1.61594959 & 221 & 0.007311989 & 6.39 & 0.0004 \\
& Total & 1.75620668 & 224 & 0.007840208 & & \\
\hline \multirow{2}{*}{$\begin{array}{l}\text { Lysiloma } \\
\text { latisiliquum }\end{array}$} & Entre grupos & 0.923860397 & 3 & 0.307953466 & & \\
& Intragrupos & 24.2889905 & 221 & 0.109904934 & 2.80 & 0.0408 \\
& Total & 25.2128509 & 224 & 0.11255737 & & \\
\hline \multirow{2}{*}{$\begin{array}{l}\text { Piscidia } \\
\text { piscipula }\end{array}$} & Entre grupos & 0.207918553 & 3 & 0.069306184 & & \\
& Intragrupos & 3.66105275 & 221 & 0.01656585 & 4.18 & 0.0066 \\
& Total & 3.86897131 & 224 & 0.017272193 & & \\
\hline
\end{tabular}

SC = Suma de cuadrados, GL = Grados de libertad, MS = Cuadrados medios, F = Valor de F; Prob > F = Nivel de confiabilidad . 
TABLA 5. Prueba de comparación múltiple de Bonferroni para categorías diamétricas de tres especies de la selva mediana subperennifolia de Quintana Roo, México.

\begin{tabular}{lcccc}
\hline \multirow{2}{*}{ Especie } & \multicolumn{4}{c}{ Categoría diamétrica } \\
\cline { 2 - 5 } & 5 & 10 & 15 & 20 \\
\hline Bursera simaruba & $0.0535 \mathrm{a}$ & $0.0764 \mathrm{a}$ & $0.0720 \mathrm{a}$ & $0.1197 \mathrm{~b}$ \\
Lysiloma latisiliquum & $0.1958 \mathrm{a}$ & $0.1848 \mathrm{~b}$ & $0.3304 \mathrm{a}$ & $0.3009 \mathrm{c}$ \\
Piscidia piscipula & $0.0949 \mathrm{a}$ & $0.1664 \mathrm{~b}$ & $0.1344 \mathrm{a}$ & $0.1679 \mathrm{c}$ \\
\hline
\end{tabular}

Valores con letras distintas son estadísticamente diferentes $(P \leq 0.05)$

TABla 6. Correlaciones paramétricas (Pearson) de los incrementos en diámetro de tres especies de la selva mediana subperennifolia de Quintana Roo, México y los elementos del clima.

\begin{tabular}{|c|c|c|c|c|c|c|c|}
\hline Especie & $\begin{array}{l}\text { Categoría } \\
\text { diamétrica }\end{array}$ & Precipitación & Sig. & Temperatura & Sig. & $\begin{array}{l}\text { Humedad } \\
\text { relativa }\end{array}$ & Sig. \\
\hline \multirow{5}{*}{ Bursera simaruba } & 5 & -0.1367 & ns & 0.6453 & ns & -0.3072 & ns \\
\hline & 10 & 0.6604 & * & 0.7699 & * & 0.2033 & ns \\
\hline & 15 & 0.3531 & ns & 0.7259 & * & 0.0362 & ns \\
\hline & 20 & 0.5804 & ns & 0.6018 & $* *$ & 0.5028 & ns \\
\hline & Todas & 0.5036 & ns & 0.8269 & $* *$ & 0.2309 & ns \\
\hline \multirow{5}{*}{$\begin{array}{l}\text { Lysiloma } \\
\text { latisiliquum }\end{array}$} & 5 & -0.1066 & ns & 0.6168 & ns & -0.0035 & ns \\
\hline & 10 & -0.0206 & ns & 0.2021 & ns & -0.102 & ns \\
\hline & 15 & 0.343 & ns & 0.5944 & ns & 0.1606 & ns \\
\hline & 20 & 0.0181 & ns & 0.6366 & ns & -0.1823 & ns \\
\hline & Todas & 0.1159 & ns & 0.7018 & * & -0.0458 & ns \\
\hline \multirow{5}{*}{ Piscidia piscipula } & 5 & 0.123 & ns & 0.822 & $* *$ & -0.2145 & ns \\
\hline & 10 & 0.5791 & ns & 0.2869 & ns & 0.7437 & * \\
\hline & 15 & 0.3307 & ns & 0.8154 & ** & -0.0347 & ns \\
\hline & 20 & 0.4816 & ns & 0.8754 & ** & -0.0352 & ns \\
\hline & Todas & 0.5472 & ns & 0.9637 & $* * *$ & 0.2304 & ns \\
\hline
\end{tabular}

Sig. Significancia; ${ }^{* * *} P \leq 0.001 ;{ }^{* *} P \leq 0.01 ;{ }^{*} P \leq 0.05 ; n s=$ no significativo

La precipitación y la humedad relativa solo se correlacionaron con el grosor del fuste de la categoría diamétrica 10 en Bursera simaruba y Piscidia piscipula, respectivamente. La temperatura fue el elemento que presentó correlaciones positivas y significativas para las tres especies, principalmente con Piscidia piscipula y Bursera simaruba (Tabla 6).

\section{DISCUSIÓN}

$\mathrm{El}$ orden de los incrementos entre especies en el presente estudio coincidió con lo encontrado por Mize et al. (1997) en Campeche (1000 mm/año - $1200 \mathrm{~mm} /$ año) donde Lysiloma latisiliquum fue la especie con mayor aumento en el grosor de su fuste y Bursera simaruba con el menor. Dicho estudio se realizó en rodales de diferentes edades (18 años, 24 años, 29 años, 30 años y > 30 años) de una selva secundaria subperennifolia con tipos de suelo similares (litosoles, rendzinas, luvisoles) a los del presente trabajo.

En la investigación de Mize et al. (1997), las tasas anuales de crecimiento también fueron superiores (B. simaruba: 0.38 $\mathrm{cm}-0.61 \mathrm{~cm} ;$ L. latisiliquum: $0.78 \mathrm{~cm}-1.0 \mathrm{~cm} ; P$. piscipula: $0.40 \mathrm{~cm}-0.61 \mathrm{~cm})$ a las encontradas en el presente trabajo. Baker et al., (2003) mencionan que diferencias entre sitios son resultado de una serie concatenada de hechos como el uso de recursos abióticos por parte de la especie, el clima, 
la apertura y tamaño de claros, así como el vigor genético de cada población.

El comportamiento de B. simaruba en el presente estudio coincide con lo mencionado por López-Ayala et al. (2006) en una selva mediana subcaducifolia de Colima, México respecto al patrón uniforme de la curva de crecimiento y a la relación del aumento en grosor del fuste en función del tamaño de los individuos. Hulshof, Stegen, Swenson, Enquist y Enquist (2012) mencionan que en B. simaruba las diferencias de crecimiento entre tamaños se deben a la disponibilidad de recursos abióticos interanuales y a la variabilidad entre micrositios.

En el caso del sitio de estudio, las bajas tasas de crecimiento - principalmente de B. simaruba - corresponden al de un bosque con sucesión secundaria cuya densidad de individuos requiere de tratamientos silvícolas de liberación que favorezcan la apertura del dosel, debido a ello es que los árboles de la mayor categoría tienen incrementos destacados ya que son aquellos que han alcanzado el dosel.

Lo mencionado por López-Ayala et al. (2006), respecto al papel del clima en el crecimiento de B. simaruba, no coincide con este trabajo debido a que en Colima se encontraron correlaciones negativas con la temperatura y positivas con la precipitación, sin llegar a ser significativos ambos casos. Valdez-Hernández, Andrade, Jackson y Rebolledo-Vieyra (2010), en una selva baja caducifolia de Yucatán, encontraron que B. simaruba presentó sus mayores intensidades de foliación en la época de secas y lluvias, coincidiendo con el aumento de la temperatura. Lo anterior permite inferir que la presencia de follaje permite actividad fotosintética que induciría la activación del cambium vascular y por ende un aumento en el diámetro (Pallardy, 2008).

Los trabajos de López-Torres y Tamarit-Urias (2005) en Campeche (1390 mm año $\left.{ }^{-1}\right)$ y de Negreros-Castillo y Martínez-Salazar (2011) en Quintana Roo (1300 mm año-1) registraron tasas de crecimiento en diámetro de $L$. latisiliquum mayores $(0.51 \mathrm{~cm} /$ año y $0.41 \mathrm{~cm} /$ año, respectivamente) a los del presente trabajo. Ambas investigaciones demostraron que las tasas de crecimiento están en función del tamaño progresivo del DAP, lo cual coincide parcialmente con esta investigación. La evidencia científica permite establecer que árboles que se encuentran en una fase de curva ascendente y que no han llegado a la etapa de plena madurez pueden presentar variación entre sus categorías diamétricas, respecto a sus tasas de crecimiento (Vanclay, 1995).

La relación del clima con el crecimiento de Lysiloma sp. fue estudiado por Daubenmire (1972), quien únicamente señalo que no había decrementos en patrón radial por efecto de la precipitación, por lo que este trabajo proporciona un mayor acercamiento sobre la influencia de la temperatura. Valdez-Hernández et al. (2010) encontraron, en dos especies de Fabaceas (Acacia gaumeri S. F. Blake, Apoplanesia paniculata C. Presl.), un patrón de foliación coincidente con el termoperiodo de la zona, lo que muestra la importancia de fenología en el proceso de crecimiento.

Los incrementos de $P$. piscipula en el presente estudio difieren de lo mencionado por Verduzco (2016) en Yucatán, donde se menciona que no se presentaron diferencias significativas entre dos categorías diamétricas (juveniles: $5 \mathrm{~cm}-10 \mathrm{~cm}$, maduros DAP $>20 \mathrm{~cm}$ ). Baker, Swaine y Burslem (2003) sugieren que variables como el micrositio y el uso particular de recursos por parte de la especie definen los patrones de crecimiento.

De acuerdo con los resultados de Verduzco (2016), P. piscipula engrosó su fuste en función de un gradiente de precipitación distribuido en tres sitios $(677.9 \mathrm{~mm}, 1132.0$ mm y $1439.6 \mathrm{~mm}$ ) lo cual no coincide con lo encontrado en Felipe Carrillo Puerto, Quintana Roo. La evidencia ecofisiológica sobre la especie en la Península de Yucatán menciona que $P$. piscipula ejerce una estrategia oportunista en el aprovechamiento del agua, con lo cual mantiene el potencial hídrico de forma constante durante la estación seca (Estrada-Medina et al., 2013), lo cual permite inferir que la temperatura es un elemento climático simultáneo en la activación de la actividad del cambium.

\section{CONCLUSIONES}

Los patrones de crecimiento de las tres especies en el ejido Andrés Quintana Roo fueron distintos y solo en una de ellas estuvo en función del tamaño de los árboles medidos 
(Bursera simaruba). Las tres especies crecen influidas por la dinámica de la temperatura.

\section{RECONOCIMIENTOS}

A la Organización de Ejidos Productores Forestales de la Zona Maya SC por su disposición y apoyo para el trabajo de campo. A la directiva ejidal de Andrés Quintana Roo, Q. R. por la dispocisión para realizar el estudio. Al Conacyt por el apoyo a R. Jabin Carreón para cursar el posgrado en Ciencias Forestales. Al Servicio Meteorológico Nacional por proporcionar los datos climáticos. A los revisores asignados por Madera y Bosques por las sugerencias al escrito.

\section{REFERENCIAS}

Baker, T. R., Swaine, M. D., \& Burslem, D. F. (2003). Variation in tropical forest growth rates: combined effects of functional group composition and resource availability. Perspectives in Plant Ecology, Evolution and Systematics, 6(1-2), 21-36.

Cattelino, P. J., Becher C. A., \& Fuller, L. G. (1986). Construction and installation of homemade dendrometer bands. Northern Journal of Applied Forestry, 3, 73-75.

Daubenmire, R. (1972). Phenology and other characteristics of tropical semi-deciduous forest in north-western Costa Rica. The Journal of Ecology, 60(1), 147-170.

Ellis, E. A., Hernández G., U., \& Romero-Montero, J. A. (2017). Los procesos y causas del cambio en la cobertura forestal de la Península Yucatán, México. Revista Ecosistemas, 26(1), 101-111. doi: 10.7818/ECOS.2017.26-1.16.

Estrada-Medina, H., Santiago, L. S., Graham, R. C., Allen, M. F., \& Jiménez-Osornio, J. J. (2013). Source water, phenology and growth of two tropical dry forest tree species growing on shallow karst soils. Trees, 27(5), 1297-1307. doi 10.1007/s00468-013-0878-9.

Fahey, R. T., \& Lorimer, C. G. (2013). Restoring a midtolerant pine species as a component of late-successional forests: Results of gap-based planting trials. Forest Ecology and Management, 292, 139-149. doi: 10.1016/j.foreco.2012.12.026.

Fassnacht, K. S., Bronson, D. R., Palik, B. J., D'Amato, A. W., Lorimer, C. G., \& Martin, K. J. (2015). Accelerating the development of old-growth characteristics in second-growth northern hardwoods. Department of Agriculture, Forest Service, Northern Research Station.
García, M. E. (1987). Modificaciones al sistema de clasificación climática de Köppen. Cuarta edición. México, D.F. 217 p.

García, C. X., \& Rodríguez, S. B. (1993). Cortas intermedias en sitios de vegetación secundaria. Ciencia Forestal en México, 18(74), 81-100.

Gotelli, N. J., \& Ellison, A. M. (2004). A primer of ecological statistics. Sunderland, Massachusetts: Sinauer Associates.

Guerra, C. W., Cabrera, A., \& Fernández, L. (2003). Criterios para la selección de modelos estadísticos en la investigación científica. Revista Cubana de Ciencia Agricola, 37(1), 3-10.

Hulshof, C. M., Stegen, J. C., Swenson, N. G., Enquist, C. A., \& Enquist, B. J. (2012). Interannual variability of growth and reproduction in Bursera simaruba: the role of allometry and resource variability. Ecology, 93(1), 180-190.

Hyams, D. (2005). Curve Expert Version 1.37. A comprehensive curve fitting package for Windows.

Interián-Ku, V. M., Vaquera-Huerta, H., Valdez-Hernández, J. I., García-Moya, E., \& Romero-Manzanares, A. (2014). Influencia de factores morfológicos y ambientales sobre el crecimiento en diámetro de Caesalpinia gaumeri Greenm en un bosque tropical caducifolio, en México. Revista Chapingo. Serie ciencias forestales y del ambiente, 20(3), 255-270.

Organización Mundial de las Naciones Unidas para la Agrigultura y la Alimentación [FAO] (2007). Base Referencial Mundial del Recurso Suelo. Primera actualización 2007. Informes sobre Recursos Mundiales de Suelos No. 103. FAO. Roma: FAO.

Kiviste, A., González, J. G. Á., Alboreca, A. R. y González, A. D. R. (2002). Funciones de crecimiento de aplicación en el ámbito forestal. Madrid, España: Instituto Nacional de Investigación y Tecnología Agraria y Alimentaria.

López-Ayala, J. L., Valdez-Hernández, J. I., Terrazas, T., \& Valdez-Lazalde, J. R. (2006). Crecimiento en diámetro de especies arbóreas en una selva mediana subcaducifolia en Colima, México. Agrociencia, 40(1), 139-147.

López-Torres, J. L., \& Tamarit-Urias, J. C. (2005). Crecimiento e incremento en diámetro de Lysiloma latisiliquum (L.) Benth. en bosques secundarios en Escárcega, Campeche, México. Revista Chapingo. Serie Ciencias Forestales y del Ambiente, 11(2), 117-123.

Lloyd, J., \& Farquhar, G. D. (2008) Effects of rising temperatures and $[\mathrm{CO} 2]$ on the physiology of tropical forest trees. Pbilosophical Transactions of the Royal Society B-Biological Sciences, 363(1498), 1811-1817. 
Miranda, F., \& Hernández, E. (1963). Los tipos de vegetación de México. Boletín de la Sociedad Botánica de México, 28, 29-179.

Mize, C., Centeno, R., \& Negreros-Castillo, P. (1997). Crecimiento de ocho especies forestales en selvas secundarias de Campeche, México. Revista Forestal Centroamericana, 19, 26-31.

Negreros-Castillo, P., \& Martínez-Salazar, I. (2011). Crecimiento y regeneración avanzada de Lysiloma latisiliquum (L.) Benth en una selva de Quintana Roo. Revista Mexicana de Ciencias Forestales, 2(5), 15-27.

Negreros-Castillo, P., \& Mize, C. W. (2013). Soil-site preferences for mahogany (Swietenia macrophylla King) in the Yucatan Peninsula. New Forests, 44(1), 85-99.

Negreros-Castillo, P., Cámara-Cabrales, L., Devall, M. S., Fajvan, M. A., Mendoza-Briseño, M.A. Mizey, C. W., \& NavarroMartínez. A. (2014). Silvicultura de las selvas de Caoba en Quintana Roo, México. Comisión Nacional Forestal. Guadalajara, México.

Organización de Ejidos Productores Forestales de la Zona Maya, S.C. [Oepfzm] (2000). Informe de paso de año 2000 del ejido Cafetal Limones. Felipe Carrillo Puerto, Quintana Roo. México. Dirección Técnica Forestal, Q. R., México.

O’Connor, C. D., Lynch, A. M., Falk, D. A., \& Swetnam, T. W. (2015). Post-fire forest dynamics and climate variability affect spatial and temporal properties of spruce beetle outbreaks on a Sky Island mountain range. Forest Ecology and Management, 336, 148-162. doi: 10.1016/j.foreco.2014.10.021.

Pallardy, S. 2008. Physiology of woody plants. New York, USA: Elsevier Inc.

Pennington, T. D., \& Sarukhán J. (2005). Árboles tropicales de México: Manual para la identificación de las principales especies. Texto Científico Universitario. Tercera edición. Fondo de Cultura Económica. Universidad Nacional Autónoma de México. México, D.F. 523 p.

Pozo, C., Armijo-Canto, N., \& Calmé, S. (2011). Riqueza Biológica de Quintana Roo. Un análisis para su conservación, vol. 2. México, D.F.: Conabio.

Ricker, M., Gutiérrez-García, G., \& Daly, D. C. (2007). Modeling long-term tree growth curves in response to warming climate: test cases from a subtropical mountain forest and a tropical rainforest in Mexico. Canadian Journal of Forest Research, 37(5), 977-989. doi: 10.1139/X06-304.
Snook, L. K. (2005). Aprovechamiento sostenido de caoba en la Selva Maya de México De la conservación fortuita al manejo sostenible. Recursos Naturales y Ambiente 44, 9-18.

Stata Corp. (2001). Stata Statistical Software: Release9.1. Stata corporation, College Station, Texas.

Toledo, M., Poorter, L., Peña-Claros, M., Alarcón, A., Balcázar,

J., Leaño, C., \& Bongers, F. (2011). Climate is a stronger driver of tree and forest growth rates than soil and disturbance. Journal of Ecology, 99(1), 254-264. doi: 10.1111/j.13652745.2010.01741.x

Valdez-Hernández, M., Andrade, J. L., Jackson, P. C., \& Rebolledo-Vieyra, M. (2010). Phenology of five tree species of a tropical dry forest in Yucatan, Mexico: effects of environmental and physiological factors. Plant and Soil, 329(1-2), 155-171.

Vanderwel, M. C., Zeng, H., Caspersen, J. P., Kunstler, G., \& Lichstein, J. W. (2016). Demographic controls of aboveground forest biomass across North America. Ecology letters, 19(4), 414-423. doi: 10.1111/ele.12574.

Vanclay, J. K. (1995) Growth models for tropical forest: A synthesis of models and methods. Forest Science, 41, 7-42.

Verduzco S., O. E. (2016). Crecimiento basal de especies arbóreas de la Peninsula de Yucatán de acuerdo a un gradiente en la disposición de agua (Tesis de maestría). El Colegio de la Frontera Sur.

Way, D. A., \& Oren, R. (2010). Differential responses to changes in growth temperature between trees from different functional groups and biomes: a review and synthesis of data. Tree Physiology, 30(6), 669-688. doi: 10.1093/treephys/tpq015.

Yáñez-Espinosa, L., Terrazas, T., \& López-Mata, L. (2006). Integrated analysis of tropical trees growth: a multivariate approach. Annals of Botany, 98(3), 637-645. doi: $10.1093 / \mathrm{aob} / \mathrm{mcl} 142$.

Manuscrito recibido el 19 de julio de 2017

Aceptado el 11 de junio de 2018

Publicado el 31 de mayo de 2019 
Pineda-Herrera et $\boldsymbol{a l}$. Crecimiento en diámetro de tres especies arbóreas en una selva secundaria

Este documento se debe citar como:

Pineda-Herrera, E., Carreón-Santos, R. J., Valdez-Hernández, J. I., \& Interián-Ku, V. M. (2019). Crecimiento en diámetro de tres especies arbóreas en una selva secundaria de Quintana Roo, México. Madera y Bosques, 25(1), e2511601. doi: 10.21829/myb.2019.2511601

Madera y Bosques por Instituto de Ecología, A.C. se distribuye bajo una Licencia Creative Commons Atribución-NoComercial-Compartirlgual 4.0 Internacional. 